\title{
EDUCACIÓN E INVESTIGACIÓN Y DESARROLLO EN AMÉRICA LATINA: LOS ÚLTIMOS TREINTA AÑOS
}

\author{
Flora Eugenia Salas Madriz \\ Docente de la Escuela de Administración Educativa \\ de la Universidad de Costa Rica
}

Recibido 11-IX-2007 • Aceptado 13-XI-2007 • Corregido 20-II-2008

\begin{abstract}
Resumen: El articulo ofrece una perspectiva general de la relación entre educación y la Investigación y el Desarrollo $(I+D)$ en América Latina y en Costa Rica en los últimos treinta años. Se hace una introducción general a las características y desarrollo de la ciencia y la tecnología en la región, se revisa el nivel $y$ avance de la investigación educativa. Se analizan sus implicaciones en el incremento de la brecha de productividad y la brecha digital en el contexto de la Globalización y la Sociedad del Conocimiento y la Información.
\end{abstract}

Palabras clave: $I+D$ en América Latina, investigación educativa en América Latina, educación y brecha de productividad, educación y brecha digital.

\section{Introducción}

En América Latina la ciencia y la tecnología se caracterizan por su bajo nivel relativo en todos los indicadores mundiales utilizados para medir su desarrollo. El gasto en actividades de ciencia y tecnología en los países latinoamericanos alcanza poco menos de los 8,000 millones de dólares anuales, lo cual representa el 2,3\% del gasto mundial en el sector (Vaccarezza, 1998, p. 2). Si bien en la década de 1990 se incrementó en la región el gasto en ciencia y tecnología, que ascendió en términos absolutos a 3,400 millones de dólares, ello sólo equivale a la mitad de lo que invierte la compañía General Motors en I+D. Para los latinoamericanos, los gastos en ciencia y tecnología representan menos del 0,5\% promedio del PIB, mientras los países desarrollados se encuentran entre el $2 \%$ y el 3\% del PIB en la mayoría de los casos.

Un rasgo característico de la investigación científica en América Latina es su gran dependencia del Estado, tanto en financiamiento como respecto de las líneas de investigación. En la región, el Estado aporta el $70 \%$ de la inversión en ciencia y tecnología. En los países desarrollados ocurre lo contrario, ya que el sector privado 


\begin{abstract}
This paper offers a general overview of the relationship between education and $R \& D$ in Latin America and Costa Rica during the last 30 years. A general introduction to the characteristics and development of science and technology in the region is made, including the level and progress of the educational research. Its implications in the increase of the productivity gap and the digital gap in the context of Globalization and the Society of Knowledge and Information are analyzed.
\end{abstract}

Key words: $R \& D$ in Latin America, educational research in Latin America, education and productivity gap, education and digital gap. asume el 90\% de los costos de la investigación (Vaccarezza, 1998). Los escasos resultados en generación de nuevo conocimiento en la región, en todos los campos científicos muestra la enorme brecha que la separa de los países productores de ciencia y tecnología del primer mundo.

Durante la "década perdida" de 1980, debido a la reducción en el gasto público la investigación en América Latina se vio severamente restringida. En la década de 1990, se iniciaron esfuerzos en la región por reactivar la investigación y el desarrollo de la ciencia y la tecnología. Desde entonces, algunos países han implementado programas sistemáticos de vinculación entre los centros de I+D públicos, especialmente universitarios, y las empresas privadas, creando parques tecnológicos, incubadoras de empresa, organismos de vinculación y transferencia, facilidades para la firma de contratos de investigación, financiamiento de innovación y riesgo compartido, entre otros. A pesar de este esfuerzo, los resultados no han sido significativos a la fecha.

En general, en América Latina la ciencia y la tecnología se caracterizan por:

- Su escasa magnitud.

- Su desvinculación con la sociedad.

- Su dependencia de la actividad científica realizada en el mundo desarrollado.

Esas características de la investigación en la región tienen como consecuencia el aumento de la brecha científica y tecnológica que impide un desarrollo económico, social y cultural coherente con las nuevas características de la economía mundial. El déficit de producción de conocimiento científico en América Latina es de grandes proporciones, porque no sólo se hace poca investigación en la región, sino que la que se hace es de bajo impacto. El conocimiento científico que se produce tiene poca o ninguna relación con la realidad social, económica y cultural de nuestros países, razón por la cual no sólo está desvinculada, sino que 
también pierde legitimidad. Así las cosas, resulta claro que para nuestras sociedades no tiene sentido invertir recursos financieros escasos en procesos de investigación de poco o ningún impacto.

Debido a la incapacidad administrativa y técnica del Estado y de las universidades públicas latinoamericanas para gestionar adecuadamente los escasos recursos para investigación, organismos internacionales como la OEA, la UNESCO, el Banco Mundial y el Banco Interamericano de Desarrollo han tratado de impulsar la investigación en la región; sin embargo, los resultados siguen siendo muy limitados con respecto a las necesidades y a sus costos reales.

Algunos críticos de la investigación en América Latina (Vaccarezza, 1998; Shiefelbein, 1995; Abraham \& Rojas, 1997) proponen que lejos de lograr desarrollo y autonomía, lo que se da en la región es una conjunción de factores que condenan a nuestros países al atraso científico y tecnológico. A la escasez de recursos económicos y humanos para la investigación, se suman el aumento de una profunda dependencia cultural y problemas estructurales y políticos, que conducen al subdesarrollo en ciencia y tecnología.

Pese a las limitaciones apuntadas, en América Latina hay grupos de investigación competitivos en el nivel internacional. También, hay esfuerzos importantes en la región en relación con la investigación en el ámbito de la educación no formal, especialmente en el campo de la educación de personas adultas, consecuencia del Plan Iberoamericano de Alfabetización y Educación Básica de Personas Jóvenes y Adultas 2007-2015.

\section{Desarrollo, investigación y educación en América Latina en la década de 1990}

En la década de 1990, debido al impacto de la Globalización, al escaso financiamiento estatal para la investigación y al surgimiento de la Sociedad del Conocimiento y la Información ${ }^{1}$, la región ingresó en una situación crítica, donde las economías vulnerables y la ingobernabilidad $^{2}$ sólo son síntomas de crisis aún mayores (Vaccarezza, 1998). Ya en el siglo XXI, América Latina presenta un balance general alarmante. "Apenas el 3\% de crecimiento del producto, sólo un punto más que durante la "década perdida" de los ' 80 ; 44\% de la población viviendo en condiciones de pobreza (CEPAL, 1999), 19\% en condiciones de indigencia y con la peor distribución del ingreso entre las regiones del mundo" (Brunner, 2000, p. 3).

Aunado a los viejos problemas, aún no resueltos, están los nuevos desafíos de la era de la Globalización y de la Sociedad del Conocimiento y la Información. Entre ellos se subrayan la revolución tecnológica, la economía informal, los mercados negros y la cultura del crimen, que abren nuevas posibilidades para el incremento del narcotráfico, la corrupción pública y privada, los delitos violentos en las zonas urbanas, la trata de personas, la prostitución y la pornografía, entre otros.

Gran parte de la desigualdad que se observa en América Latina es consecuencia de las pronunciadas diferencias salariales entre los distintos sectores de la población, que reflejan una distribución desigual en la cantidad y la calidad de la educación. Esa desigualdad también pone en evidencia diferencias de género, brechas entre el empleo formal e informal, entre ingresos rurales y urbanos, y otras formas de segmentación del mercado laboral (Mesén, 2003).

La educación inequitativa y de baja calidad en América Latina es considerada como una de las principales causas de la desigualdad en la región. Se ha comprobado que las diferencias en la educación se transmiten de una generación a otra, a través de la familia, lo que genera un "círculo de la pobreza", en el que quedan atrapadas millones de personas, especialmente jóvenes en edad productiva (Mesen, 
2003). En ese contexto, la investigación en el campo de la educación se vuelve crucial, ya que constituye uno de los ejes fundamentales para resolver en parte algunos de los principales problemas sociales, económicos y culturales de la región.

La educación es el principal recurso productivo con el que cuentan la mayoría de las personas y esto aplica para el 10\% más rico de la población. Se ha encontrado que lo que determina las condiciones y calidad de vida de ese segmento es su nivel educativo y, en consecuencia, las características de sus empleos, la zona de residencia y el número de hijos. Estas personas suelen desempeñarse en cargos directivos, profesionales, técnicos, como empresarios o funcionarios superiores de empresas públicas y privadas. Sus ingresos les permiten acceder a viviendas con todos los servicios, seguras y salubres, por lo general ubicadas en zonas urbanas. Suelen tener dos o menos hijos y tienen condiciones para ahorrar y enfrentar situaciones de enfermedad, invalidez, jubilación y muerte (Mesén, 2003, pp. 20-22).

En contraste, los deciles más pobres de la población, al no acceder a la educación y contar con muy baja escolaridad, sólo logran emplearse en actividades poco remuneradas o en negocios informales, suelen vivir en zonas rurales o urbanomarginales, y tienen en promedio entre 3,5 a 4 hijos, variando de país en país en América Latina (Mesén, 2003). En ese contexto, “... las desigualdades en la distribución del ingreso en los países latinoamericanos están relacionadas con características que distinguen a los grupos de más altos ingresos de los demás. Las brechas en la cima de la distribución, más que las diferencias entre los grupos medios o pobres, son las que hacen de América Latina la región más desigual del mundo" (Mesén, 2003, p. 24).

En un marco social de inequidad, la educación en América Latina, a pesar de todos los esfuerzos por reformarla y elevar su calidad, continúa presentando serias debilidades. Si bien el gasto público en educación aumentó en la región aproximadamente un $22 \%$ entre 1990 y 1996 , para continuar incrementándose hasta finales de esa década, en los comienzos del siglo XXI persisten viejos problemas que se suman a los nuevos:

Cobertura insuficiente e importantes niveles de exclusión en algunos países; escasa capacidad de compensar los déficit de capital cultural de los niños y jóvenes provenientes de los hogares más pobres; altas tasas de repitencia y deserción que se traducen en un elevado grado de desperdicio (UNESCO, 1998); resultados insatisfactorios de aprendizaje (UNESCO, 1998a); recursos humanos y materiales limitados y habitualmente mal gestionados y/o utilizados dentro del sistema educacional; débil articulación entre niveles del sistema y de éste con el entorno, particularmente el mercado de trabajo; mediocre nivel educacional de la fuerza de trabajo mayor de 25 años (5 años en promedio) y un $14 \%$ de la población sumida en el analfabetismo; pronunciadas diferencias en cuanto a años de escolarización de la población entre el $10 \%$ más rico (11 o más años) y el 30\% más pobre (menos de 4 años); escasa producción de conocimientos para mejorar los procesos de enseñanza y aprendizaje que permita alimentar las políticas públicas destinadas a este sector, etc. (Brunner, 2000, p. 3).

Con los indicadores de desarrollo educativo que registra América Latina es indispensable aumentar la investigación en el campo de la educación que ofrezca conocimientos sobre los procesos mismos de enseñanza y orienten la toma de decisiones y la formulación de políticas en la región. En la actualidad no se puede eludir que existe una fuerte presión de la población por educación accesible, de calidad y pertinente:

La gente manifiesta una creencia cada vez más amplia respecto al papel que la educación desempeña en las actuales condiciones de la sociedad para prosperar materialmente y ganar movilidad social. Así, por ejemplo, la última versión del Latinbarómetro (2000), muestra que en América Latina (17 países) un $60 \%$ de la población estima que "lo más importante en la vida para tener éxito es la educación", contra un $19 \%$ que elige en vez el "trabajo duro", un $12 \%$ "las conexiones" y un $8 \%$ "la suerte" (Brunner, 2000, p. 4). 


\section{Los desafíos en $I+D$ en América Latina en la década de 2000}

Como respuesta a los retos de la Globalización $^{3}$, a mediados de la década de 1990 hubo cambios en las políticas regionales de ciencia y tecnología inducidos por organismos internacionales como la CEPAL, el SELA, el BID y la OEA. Estos cambios se orientaron al mejoramiento de la gestión de los sistemas de ciencia y tecnología, y a la consolidación de un mayor apoyo financiero del Estado para la I+D. Mientras en 1990 se contaba en América Latina con 164,677 investigadores, para 2002 se tenían 248,109. En 1990 el aporte del presupuesto estatal fue de un $0,49 \%$, y para 2001 sólo subió a un $0,64 \%$ en la región. Ese aumento es importante pero insuficiente, si se le compara con la inversión que hicieron en $\mathrm{I}+\mathrm{D}$ los gobiernos de Estados Unidos $(2,64 \%)$ y Canadá $(1,88 \%)$ en 2001. Pese a ello, el leve incremento en la limitada inversión estatal en $\mathrm{I}+\mathrm{D}$ tiene consecuencias directas en la producción científica de América Latina, debido a que:

Este crecimiento no se destaca sólo en el gasto en investigación y desarrollo, sino en los resultados más importantes como las publicaciones y solicitudes de patentes, de hecho, se publicaron en Sciencie Citation Index, 11.046 artículos para el año de 1990, en comparación con los 35.299 artículos publicados en el año 2003 por América Latina. Por otro lado, del total de patentes solicitadas para el año de 1990 estimadas en 25.468, en el 2002, esta cifra se duplicó al sumar 55.286 patentes requeridas por los países de la región. (RICYT, 2005) (Royero, 2005, s. n. p.).

Los datos sobre I+D de América Latina, Estados Unidos y Canadá, revelan aspectos interesantes sobre el desarrollo de la ciencia y la tecnología en la región. Mientras en Estados Unidos y Canadá el sector empresarial duplica la inversión estatal en $\mathrm{I}+\mathrm{D}$, en América Latina ocurre el fenómeno inverso, ya que es el Estado el que invierte la mayor cantidad de recursos en desarrollo de la ciencia y la tecnología.

Las universidades juegan un papel importante en América Latina en I+D, mientras que ocurre lo contrario en los Estados Unidos y Canadá. Si bien la inversión en desarrollo de conocimiento científico y tecnológico de esas instituciones es pequeña en comparación con la del Estado, hacen un aporte significativo a la generación de conocimiento en la región. Aumentar el apoyo financiero del Estado y del sector privado a las universidades para I+D es una estrategia que favorece el desarrollo de la ciencia y la tecnología en la región.

\section{Cuadro $\mathrm{N}^{\circ} 1$}

Gasto en I+D por sector de financiamiento en América Latina vs. países desarrollados (USA, Canadá) para el año 2002

\begin{tabular}{lccc}
\hline Gasto por sector & $\begin{array}{c}\text { América } \\
\text { Latina }\end{array}$ & USA & Canadá \\
\hline Gobierno & $56,9 \%$ & $30 \%$ & $24,9 \%$ \\
Empresas & $37,2 \%$ & $64,6 \%$ & $45,3 \%$ \\
Educación Superior & $4,4 \%$ & $2,7 \%$ & $\cdots$ \\
Organizaciones privadas sin fines de lucro & $0,4 \%$ & $2,7 \%$ & $2,7 \%$ \\
Extranjero & $1,1 \%$ & $\cdots$ & $12,2 \%$ \\
\hline
\end{tabular}

Fuente: Royero, 2005. 


\subsection{I+D en Educación en América Latina}

La investigación educativa en la región tuvo un desarrollo importante en la década de 1960, porque los gobiernos latinoamericanos estaban interesados en mejorar el sistema educativo público y financiaron investigación orientada a determinar los sectores vulnerables de la población y las condiciones para favorecer el desarrollo de la educación primaria y secundaria.

En la década de 1970, la investigación educativa tuvo un enorme impacto de las propuestas teórico-metodológicas de Paulo Freire, por lo que predominaron investigaciones reflexivas de corte participativo. El objetivo de la investigación en ese período fue, primordialmente, aproximarse a la realidad social, captar su dinámica desde adentro y transformarla, partiendo del supuesto de que la educación tiene una función liberadora y está al servicio de los intereses de las personas, y no de las clases favorecidas ni de las empresas nacionales y extranjeras explotadoras de la mano de obra barata.

En la década de 1980 predominaron los enfoques críticos sobre la investigación convencional. Se cuestionó el modelo positivista y las concepciones de la denominada "tecnología educativa", lo que condujo a explorar nuevas formas de acercamiento a la realidad educacional, en particular en la escuela. En la década de 1990 surgieron nuevas líneas de investigación para responder a los problemas que afectaban a la educación en ese período. Por ello, se iniciaron esfuerzos en la región para dar respuesta a las dificultades de la educación pública en la Era de la Globalización.

En ese escenario aparece el interés por el estudio de las nuevas tecnologías de la información y la comunicación (TIC). También en esa década, en el marco de las reuniones del Comité Regional Intergubernamental del Proyecto Principal de Educación en América Latina y el Caribe, y de las últimas reuniones de
Ministros de Educación de la región, se plantearon temas prioritarios a investigar, que debían ser impulsados y apoyados por los diferentes países. Entre ellos:

- La búsqueda de consenso en la definición de políticas.

- Innovaciones para renovar los procesos pedagógicos.

- La exploración de nuevas estrategias de formación docente.

- $\quad$ Avances en cobertura, calidad y equidad.

- $\quad$ El incremento de la investigación e información para la toma de decisiones políticas.

Como consecuencia del proceso de apertura económica producto de la Globalización, hubo temas emergentes que se debían investigar a finales del siglo XX y comienzos del siglo XXI, como:

- Los procesos pedagógicos en el aula.

- Nuevas opciones educativas y pedagógicas en un mundo cada vez más globalizado y con acceso a niveles crecientes de información mediada por las nuevas tecnologías de la información y la comunicación (TIC's).

- Cambios relevantes en los procesos de enseñanza y aprendizaje y en la administración educativa (Abraham \& Rojas, 1997).

Algunos especialistas señalan que en América Latina “...no se ha dedicado suficiente tiempo a la identificación, la comprensión y la definición de los problemas clave de la educación, en especial a los que se presentan en la sala de clase en los niveles de educación primaria y secundaria y a aquellos que se refieren a desarrollar la tradición de la investigación empírica a nivel universitario" (Schiefelbein, 1995, p. 22).

Otro aspecto indispensable para superar la crisis en la que se encuentra la educación en la región es la superación del sistema educativo "frontal" o "tradicional", 
e implementar nuevas estrategias pedagógicas en las que se enfatice “... una educación centrada en el alumno -lo que no es algo nuevo en educación-, y su rol activo para construir el significado" (Schiefelbein, 1995, p. 19), donde el profesor juega un rol de mediador. Esta tesis hace referencia al cambio del paradigma educativo conductista al constructivista.

Pese a que la educación es un área crítica para el desarrollo de América Latina es donde menos producción científica existe hasta ahora en la región. En una investigación hecha con base en los datos de la Red Latinoamericana de Información y Documentación en Educación (REDUC), se encontró que para el período comprendido entre 1985-1995, sólo un 10\% de los resúmenes analíticos se referían a investigación. A partir de ese estudio, que incluyó el $56 \%$ de esos resúmenes analíticos, se determinó que las temáticas tratadas en investigación, en términos porcentuales, fueron las siguientes:

- Docentes (formación, capacitación, perfil del profesor, profesionalización, entre otros), con un $9,2 \%$ del total de resúmenes.

- Distintas disciplinas (Ciencias Naturales, Matemáticas, Español, entre otras), con un 7,2\% del total de resúmenes.

- Diagnósticos y otros estudios generales del sistema educativo, con un $4,7 \%$ del total de resúmenes.

- Innovación educativa, con un 4,5\% del total de resúmenes.

- Clima organizacional y de aula, con un $4,2 \%$ del total de resúmenes

a. Temas con proporciones inferiores al $4 \%$ :

- $\quad$ Alrededor del 60\% de las investigaciones se refieren a la educación formal.

- $\quad$ Alrededor de un 19\% aluden a la educación no formal (Abraham y Rojas, 1997a, 1997b).
Las temáticas que concentraron el $95,5 \%$ del total del material estudiado eran de nivel macro: sistema educativo y políticas educacionales, investigaciones históricas e investigaciones acerca de la investigación (Abraham \& Rojas, 1997). El escenario de la producción científica en educación es tan limitado y escaso como el de otras áreas básicas para el desarrollo de Latinoamérica.

En la actualidad está demostrado que el desarrollo de la ciencia y la tecnología de un país es imposible sin un sistema educativo de calidad, en el que se forme al estudiantado de primaria y secundaria en competencias cognitivas de alto nivel, que favorezcan su ingreso y permanencia en el sistema de educación superior. Los requerimientos actuales del mundo social, académico y laboral exigen tanto un buen nivel educativo, como la capacidad para continuar procesos de formación continua a lo largo de la vida. Entre las competencias básicas que debe tener un ciudadano promedio en la actualidad para acceder a condiciones de trabajo y de vida adecuadas están (Vizcarro \& León, 1998):

\section{A. Competencias cognitivas:}

- Solución de problemas

- Pensamiento crítico

- Formulación de preguntas pertinentes

- Búsqueda de información relevante

- Realización de juicios informados

- Uso eficiente de la información

- Realización de observaciones

- Capacidad para investigar

- Invención y creación

- Análisis de datos

- Presentación de trabajos y conclusiones en forma eficiente, tanto oralmente como por escrito

\section{B. Competencias metacognitivas:}

Autorreflexión

Autoevaluación 


\section{Competencias sociales:}

- Participar en actividades de grupo

- Persuadir

- Trabajar cooperativamente

\section{Disposiciones afectivas:}

- Perseverancia, motivación intrínseca, buen nivel de iniciativa y una actitud responsable, independencia, flexibilidad y capacidad para enfrentar situaciones frustrantes y cambiantes.

El desarrollo de esas competencias exige un nivel suficiente de I+D en el ámbito educativo, que promueva la calidad de la enseñanza en general y una formación universitaria de alto nivel, que ofrezca adicionalmente programas de educación continua y de reciclaje profesional para que el profesorado y el personal vinculado a la educación tengan un buen desempeño. Los datos en investigación educativa en la región muestran que ese requerimiento para garantizar y asegurar la calidad de la educación no se cumple en la región. Para Puryear (2000) los desafíos de la educación en América Latina son:

1. Un nuevo enfoque politico de la educación: Superar la visión política tradicional orientada a la extensión en la cobertura escolar y orientarse a la calidad educativa. Ese paso abriría posibilidades para una educación más pertinente y equitativa en la región.

2. En términos de acceso a la educación hay avances importantes en primaria y secundaria, pero continúa el rezago en el sector terciario, donde el porcentaje de matrícula fue en total en la región en 1960 de un 1,8\%, en 1970 de $2,8 \%$, en 1980 de $5,4 \%$, en 1990 de 6,4\% y para 1993 de un 6,6\% (Puryear, 2000). Sin el incremento en la matrícula en educación superior, no será posible superar las limitaciones y el atraso actual en ciencia y tecnología en América Latina.

3. La calidad de la educación: es un hecho que para que haya buena educación debe haber una inversión que la haga posible y sostenible. La deserción, el bajo rendimiento académico en general y en las áreas de ciencias y matemáticas en particular son consecuencia de ello. En la región:

Los gastos promedio por estudiante se mantienen muy por debajo del de los países industrializados. La crisis de la deuda de los 80 provocó que el gasto de los gobiernos en educación bajara significativamente. A nivel global, el gasto público en educación bajó, en términos reales, en un $15 \%$ entre 1980 y 1985 , en tanto que el gasto per cápita descendió cerca de $25 \%$. Un estudio al respecto determinó que los gastos promedio por estudiante en educación primaria bajaron de US \$ 164 a US\$ 118 (Wolff, Schiefelbein y Valenzuela 1993, 80-81). El gasto ha comenzado a recuperarse pero se mantiene en una pequeña fracción de gasto por alumno con respecto a los países desarrollados (Puryear, 2000, p. 21).

4. La equidad educativa: La desigualdad en los ingresos de las familias determina las desigualdades en el tipo, cantidad y calidad de educación al que tendrán acceso en sus vidas. En la región las políticas gubernamentales han inclinado la balanza para favorecer a los sectores más ricos de la población, restando recursos a los sectores más pobres; de hecho.

Estas políticas le han dado a América Latina un sistema de colegios privados de buena calidad para los estudiantes de clase media y alta y de escuelas públicas de baja calidad para los sectores más pobres. Un planificador caracteriza la presente situación como una de oferta retórica carente de recursos: "Los pobres han recibido un derecho - habrá educación universal. Pero sin recursos, la calidad de esa educación y, por consiguiente, el valor de ese derecho se ha derrumbado" (Nancy Birdsall, citado en Puryear y Olivos, 1995, 3). Cuando se recuerda que América Latina tiene la ingreso más desigual del mundo y que la educación es la variable que comúnmente se 
cree que tiene el mayor impacto en la desigualdad del ingreso, las consecuencias de mantener las políticas existentes son claras y descorazonadoras (Londoño, 1996; Edwards, 1995, pp. 252-260). (Puryear, 2000, p. 27)

\section{La estructura y organización de los sistemas educativos: Las} organizaciones burocráticas y centralizadas, de baja eficiencia y pocas condiciones para la evaluación del desempeño y la rendición de cuentas limitan el avance de los cambios e innovación educativa, porque:

Los sistemas centralizados restringen la autonomía y la transparencia de los resultados. Muchos de los problemas que afectan a las escuelas se derivan del diseño y funcionamiento de los sistemas actuales, caracterizados por burocracias centralizadas, procedimientos rígidos y aislamiento de otros sectores de la sociedad. Normalmente, los profesores son empleados por los gobiernos nacionales o provinciales en lugar de serlo por las municipalidades o los propios establecimientos.

La centralización ha significado también que los directores de escuelas públicas (en contraste con los colegios privados) tengan pocas posibilidades de manejar sus establecimientos en forma efectiva mediante la definición de presupuestos, la adaptación de insumos educativos a las condiciones locales y la selección de profesores. En la mayoría de los países, estas funciones son monopolizadas por burocracias centralizadas, limitando la responsabilidad de los rectores en el proceso educativo. Por consiguiente, las escuelas tienen pocos incentivos - y capacidad-para responder a las preocupaciones de los padres y de la comunidad (Puryear, 2000, p. 29).

\section{El deterioro de la profesión} docente: El deterioro sistemático que ha experimentado la educación pública latinoamericana desde la década de 1980 tiene como una de sus consecuencias más negativas el deterioro de la calidad de la formación del profesorado, los términos contratación, los salarios y de las condiciones de trabajo. Además, ha repercutido en políticas de recargo de funciones e impedimentos administrativos para la formación en servicio y la educación continua, que han vuelto obsoletos a muchos docentes. Para la educación en la región:

Un problema adicional es el estado lamentable de la profesión docente en América Latina. Los profesores de todos los niveles educacionales están generalmente mal formados y peor pagados y tienen pocos incentivos para la excelencia profesional y el perfeccionamiento. Un tercio de los profesores de la región carece de certificados o de grados profesionales (World Bank, 1993, p. 44). En los colegios rurales del nordeste brasileño, sólo el 40\% de los profesores han completado la enseñanza básica (Harbison y Hanushek, 1992).En México, los profesores fueron uno de los pocos grupos ocupacionales cuyos salarios se redujeron y bajaron de decil con respecto a otros durante los 80 (de Ibarrola 1995, p. 262). (Puryear, 2000, p. 30).

\section{Mantener la competitividad eco-} nómica internacional: Como se mencionó anteriormente, los requerimientos del mundo social, cultural y laboral actual rebasan en mucho las competencias de la formación tradicional, pero dar acceso a la población a las condiciones necesarias para que adquiera las nuevas competencias que exige nuestro tiempo tiene como condición superar los problemas mencionados en los puntos anteriores. De hecho:

La mayoría de los analistas están de acuerdo con que un nuevo enfoque del desarrollo educativo tiene que estar ligado a la mantención de la competitividad económica internacional. La competencia internacional exitosa requiere reforzar las destrezas tradicionales y agregar algunas nuevas, como respuesta a los cambios en el mercado de trabajo. Esto significa proveer el conocimiento, los valores y las habilidades que promuevan la innovación tecnológica. Esto, a su vez, probablemente signifique fortalecer la formación básica en lecto-escritura y matemáticas, asignándole un especial énfasis a las ciencias y desarrollando la habilidad de los estudiantes de razonar y aprender por su propia cuenta (Puryear, 2000, p. 32).

Si bien la solución a estos problemas tiene un componente económico importante, el verdadero desafío es hacer las transformaciones políticas, organizativas, institucionales y de cultura que el cambio educativo requiere. 


\section{Los desafíos en I+D en Costa Rica en la década de 2000}

Para 1996, en Costa Rica no se superaron los 500 investigadores por millón de habitantes, y la inversión en $\mathrm{I}+\mathrm{D}$ fue de un 0,5\% del PIB. Los datos de 2005 no muestran avances al respecto, ya que en el país se invirtió en ese año un 4,4\% del PIB en la educación pública, un $0,2 \%$ del PIB en I+D, y se contó con sólo 533 profesionales por millón de habitantes dedicados a la generación de conocimiento (CONARE, 2006).

Pese a las limitaciones en investigación de Costa Rica los aportes en la producción de nuevo conocimiento científico de las universidades públicas son significativos, debido a que en ellas se ejecutan la mayoría de los proyectos y programas de investigación del país. En 2004 hubo 338 investigadores de las universidades públicas por millón de habitantes, que trabajaron en 1128 proyectos y 86 programas de investigación (Montiel, 2006). Los datos muestran que la superación de la brecha del conocimiento radica, en gran medida, en la transformación de las universidades públicas, a fin de promover la pertinencia y calidad de la docencia, la calidad y cantidad de la investigación y el impacto de la acción social.

Pese a que Costa Rica es un país con mejores indicadores de desarrollo que muchos países de América Latina, los problemas que aquejan a la región se manifiestan a lo interno de una forma u otra. Como muestran los datos del cuadro 2 (Ruiz, 2006), la matrícula en primaria, secundaria y educación superior está por encima de la que presentan otros países.

Sin embargo, problemas como la deserción se han vuelto endémicos en nuestro sistema educativo público, como muestran los datos del cuadro 3 (Ruiz, 2006). La deserción escolar está asociada a otros factores. Unos son intrínsecos al sistema educativo, otros están vinculados al deterioro social, la pobreza, la desintegración familiar, las adicciones, entre otros (Ruiz, 2006).

Cuadro 2

Tasas de matriculación en algunos pises de América Latina

Año 2002

\begin{tabular}{|c|c|c|c|c|}
\hline País & $\begin{array}{c}\text { Tasa Bruta } \\
\text { Primaria }\end{array}$ & $\begin{array}{l}\text { Tasa Bruta } \\
\text { Secundaria }\end{array}$ & $\begin{array}{c}\text { Tasa Neta } \\
\text { Secundaria }\end{array}$ & $\begin{array}{c}\text { Tasa Bruta } \\
\text { Terciaria }\end{array}$ \\
\hline Guatemala & 106 & 43 & 26 & 9 \\
\hline Nicaragua & 109 & 61 & 39 & 18 \\
\hline $\begin{array}{l}\text { República } \\
\text { Dominicana }\end{array}$ & 124 & 59 & 36 & 34 \\
\hline El Salvador & 113 & 59 & 49 & 17 \\
\hline Ecuador & 117 & 59 & 50 & - \\
\hline Costa Rica & 104,9 & 65,6 & 58,7 & 23,8 \\
\hline Colombia & 111 & 71 & $54^{* *}$ & 24 \\
\hline México & 110 & $76^{* *}$ & 58 & $22^{* *}$ \\
\hline Panamá & 112 & 71 & $60 * *$ & 43 \\
\hline Perú & 120 & $89^{* *}$ & 69 & $32^{* *}$ \\
\hline Brasil & $149^{* *}$ & 108 & $72^{* *}$ & $18^{* *}$ \\
\hline Chile & 100 & 89 & 79 & 42 \\
\hline Argentina & $120 * *$ & $100 * *$ & $81^{* *}$ & $56^{* *}$ \\
\hline Honduras & $106^{* *}$ & - & - & 15 \\
\hline
\end{tabular}

Fuente: Para Costa Rica OPES-CONARE, y para el resto de países UNESCO.

** Datos corresponden al 2001. 
Cuadro 3

Porcentaje de deserción intra-anual en educción regular según nivel educativo ${ }^{\mathrm{a}}$ 1990-2004

\section{Porcentaje}

\begin{tabular}{|c|c|c|c|c|c|c|}
\hline & 1990 & 1995 & 1996 & 1997 & 1998 & 1999 \\
\hline Educación Primaria & 4,7 & 5,0 & 4,5 & 4,5 & 4,9 & 4,4 \\
\hline $1^{\circ}$ & 6,8 & 7,2 & 6,4 & 6,5 & 6,8 & 6,6 \\
\hline $2^{\circ}$ & 4,9 & 5,0 & 4,5 & 4,7 & 5,0 & 4,6 \\
\hline $3^{\circ}$ & 4,0 & 4,5 & 4,0 & 3,9 & 4,1 & 3,8 \\
\hline $4^{\mathrm{o}}$ & 3,9 & 4,6 & 4,2 & 4,3 & 5,0 & 3,9 \\
\hline $5^{\mathrm{o}}$ & 4,2 & 4,1 & 4,0 & 3,9 & 4,3 & 4,0 \\
\hline $6^{\circ}$ & 3,1 & 3,6 & 2,9 & 3,0 & 3,3 & 2,9 \\
\hline Educación Secundaria ${ }^{b}$ & 10,3 & 12,7 & 11,0 & 10,8 & 10,9 & 9,2 \\
\hline $7^{\circ}$ & 17,5 & 21,8 & 20,3 & 19,9 & 19,8 & 17,1 \\
\hline $8^{\circ}$ & 6,9 & 8,8 & 6,8 & 7,5 & 7,4 & 6,0 \\
\hline $9^{\circ}$ & 4,6 & 5,7 & 5,2 & 5,2 & 4,8 & 4,0 \\
\hline $10^{\circ}$ & 10,0 & 11,6 & 8,8 & 7,0 & 8,0 & 6,9 \\
\hline $11^{\circ}$ & 4,3 & 4,9 & 3,0 & 3,1 & 2,5 & 6,9 \\
\hline \multirow[t]{2}{*}{$12^{\circ}$} & 4,7 & 1,4 & 2,4 & 1,3 & 3,9 & 2,3 \\
\hline & 2000 & 2001 & 2002 & 2003 & 2004 & $\begin{array}{c}\text { Promedio } \\
1990-2004\end{array}$ \\
\hline Educación Primaria & 4,1 & 4,5 & 4,0 & 3,9 & 3,3 & 4,3 \\
\hline $1^{\mathrm{o}}$ & 5,9 & 6,1 & 5,3 & 5,1 & 4,1 & 6,1 \\
\hline $2^{\mathrm{o}}$ & 4,2 & 4,5 & 3,8 & 3,8 & 3,5 & 4,4 \\
\hline $3^{\circ}$ & 3,5 & 4,2 & 3,4 & 3,9 & 3,1 & 3,8 \\
\hline $4^{\circ}$ & 4,0 & 4,3 & 3,8 & 3,6 & 3,4 & 4,0 \\
\hline $5^{\mathrm{o}}$ & 3,6 & 4,4 & 3,7 & 3,6 & 3,1 & 3,9 \\
\hline $6^{\circ}$ & 3,3 & 3,2 & 3,5 & 3,4 & 2,8 & 3,1 \\
\hline Educación Secundariab & 10,2 & 11,3 & 10,8 & 9,4 & 10,3 & 10,7 \\
\hline $7^{\circ}$ & 18,6 & 19,5 & 19,1 & 16,6 & 18,3 & 19,0 \\
\hline $8^{\circ}$ & 7,9 & 9,1 & 7,8 & 6,8 & 8,5 & 7,6 \\
\hline $9^{\circ}$ & 4,4 & 4,7 & 4,7 & 4,1 & 4,0 & 4,9 \\
\hline $10^{\circ}$ & 8,0 & 10,4 & 9,7 & 8,7 & 9,4 & 9,2 \\
\hline $11^{\circ}$ & 2,7 & 3,1 & 2,6 & 3,0 & 2,9 & 3,8 \\
\hline $12^{\circ}$ & 4,3 & 3,8 & 3,9 & 5,3 & 4,6 & 3,7 \\
\hline
\end{tabular}

a/ Dependencia: pública, privada y privada-subvencionda. b/ Secundaria académica y técnica diurna.

Fuente: C. Castro, con base en Deptamento de Estadística, MEP. 
Entre los factores propios del sistema educativo están, como se señaló anteriormente: las políticas educativas, la baja inversión del Estado en educación, la estructura y cultura de la organización institucional, la formación del profesorado (inicial y en servicio), las condiciones salariales y los estímulos al personal docente (Puryear, 2000, Ruiz, 2006). Todos esos factores inciden de manera directa en la calidad, la pertinencia y la equidad de la educación.

Los principales desafíos en $\mathrm{I}+\mathrm{D}$ en Costa Rica para la década de 2000 y que probablemente persistirán en la de 2010 están asociados al mejoramiento general de la educación. La producción de conocimiento científico y de tecnología tiene como condición que haya personal altamente capacitado para realizar procesos de investigación complejos. Sin una sólida educación en primaria y secundaria, y sin un fortalecimiento significativo en la formación científica de niños y jóvenes, el ingreso a las universidades seguirá siendo limitado $\mathrm{y}$, en consecuencia, el número de personas con el perfil académico necesario para llevar a cabo investigación científica de alto nivel seguirá siendo insuficiente para abarcar la cantidad y calidad de investigación que requiere el desarrollo económico y social integral de la región.

\section{Conclusiones}

La preocupación creciente a nivel regional y mundial por la educación muestra que hay conciencia a escala planetaria de que ella constituye un medio indispensable e irremplazable para el desarrollo económico y humano. Con mucha más razón el debate educativo ha cobrado relevancia en América Latina y el Caribe, donde las nuevas tendencias de la economía de la Globalización y la Sociedad del Conocimiento y la Información han agudizado las asimetrías socioeconómicas y las brechas del desarrollo que históricamente la han separado de los países y las regiones desarrolladas (Abraham \& Rojas, 1997b).

El esfuerzo de cada país, sumado a estrategias regionales y a la cooperación internacional abrió en la década de 2000 nuevos espacios para el desarrollo de la educación, dando especial importancia a la formación inicial y en servicio de educadores y administradores de la educación. La diferencia con períodos anteriores es que en la actualidad las soluciones no son "enlatados" traídos de otras regiones, sino procesos que tienen como fundamento la investigación hecha por científicos locales y regionales y, en consecuencia, las soluciones tendrán una mayor probabilidad de ajustarse a la realidad y de responder a problemas concretos.

Elevar la capacidad de I+D en las universidades públicas es una tarea impostergable, que requiere de decisiones políticas y de recursos adicionales a los que se destina en la actualidad para tal propósito. Encontrar fórmulas alternativas de financiamiento al presupuesto ordinario es un reto que deben asumir las instituciones de educación superior pública. Esfuerzos por vincular la investigación universitaria al sector productivo son muestra de que las universidades han encontrado nuevas vías para incrementar y mejorar la cantidad, calidad y pertinencia de la investigación.

En la actualidad se debe desarrollar la investigación en educación en atención a las líneas y enfoques emergentes, más acordes con los problemas y requerimientos de conocimiento científico de la región (Abraham \& Rojas, 1997b). Un área que es crítica es la formación de formadores, tanto en el nivel inicial como en servicio, para garantizar que el sistema educativo cuente con el personal docente y administrativo idóneo para realizar las acciones de cambio e innovación hacia las que tiende la educación contemporánea. Asimismo, la formación continua es una necesidad poco atendida en el país, que exige soluciones en el corto plazo. 
Áreas de investigación fundamentales en Costa Rica son la formación en primaria y secundaria, donde el desempeño del estudiantado en lecto-escritura, matemáticas y en las ciencias, en general, es débil. Sin una sólida formación en esas áreas el estudiantado tiene pocas probabilidades de acceder a la educación superior y de mantenerse en ella hasta culminar sus estudios superiores. Sumado a lo anterior, si la formación profesional inicial es débil el estudiantado tendrá pocas probabilidades de ingresar en programas de posgrado y de especializarse en investigación.

A nuestro parecer, una de las principales limitaciones del sistema educativo costarricense, desde primaria hasta la educación superior, no son las matemáticas, el inglés o las ciencias, sino la lecto-escritura en español. Un estudio hecho hace veinte años (Rodino \& Ross, 1985), mostró que el estudiantado universitario presentaba entonces serios problemas de expresión escrita. Esos problemas son concomitantes a problemas de lectura, que si se suman son un impedimento básico para el desarrollo de habilidades de pensamiento de alto nivel.

El principal medio del pensamiento es el lenguaje (Vygotski, 1995), un mal desarrollo del lenguaje inevitablemente conlleva limitaciones de pensamiento. Con base en mi experiencia con estudiantes universitarios de grado, licenciatura y posgrado en varias universidades públicas y privadas del país, estoy convencida de que si el estudio de Rodino \& Ross (1985) se repitiera hoy, arrojaría resultados sobre el desempeño escrito del estudiantado universitario tan dramáticos, incluso más, que los de 1985.

Sin una capacidad lecto-escritora adecuada del estudiantado, la comprensión de disciplinas complejas como: las Matemáticas, la Filosofía, la Física, la Bioquímica, la Historia o la Biotecnología, no es posible. Cuando nuestros estudiantes de primaria, secundaria y de la universidad tengan un buen desarrollo del lenguaje materno y hablen, lean y escriban con propiedad en español, podremos enseñarles $-\mathrm{y}$ sin duda "aprenderán"-, otros contenidos y disciplinas, incluso Matemáticas puras, Física Cuántica y otros idiomas.

\section{Notas}

1. Los orígenes de la Sociedad de la Información y el Conocimiento se asocian a tres fenómenos, en principio autónomos e independientes: la crisis económica del capitalismo y el socialismo soviético de la década de 1980, la aparición de movimientos sociales emergentes orientados a la protección de los Derechos Humanos, el medioambiente, el feminismo, los derechos de minorías, entre otros; y la revolución de las TIC y su impacto en todas las esferas de la economía, la sociedad y la cultura. Su confluencia desencadenó un sistema de producción y reproducción social, cuya base es la generación del conocimiento, el acceso masivo a la información, la innovación tecnológica y la configuración de redes globales de almacenamiento de la información en formato digital, de las cuales la Internet es la más importante (Castells, 2001a, 2001b).

2. La ingobernabilidad es la condición de crisis generalizada del modelo de gobierno de Estado democrático, provocada por el desmantelamiento del Estado Benefactor y el subsecuente desequilibrio entre el volumen de pretensiones y demandas sociales y la capacidad del gobierno para satisfacerlas. En Costa Rica esa crisis se hizo presente a principios de la década de 1990, producto del desmantelamiento del Estado Benefactor que se dio en la década de 1980 (Salas, 1993).

3. Proceso en el cual los Estados nacionales se diluyen a través de actores transnacionales y sus lógicas de poder, orientación económica, identidad y cultura. Entre ellos, destacan los mercados y el comercio internacional, así como el predominio de la lógica económica liberal. Según U. Beck (1988), este movimiento es irreversible y se caracteriza por: “... la creciente densidad del intercambio internacional, la revolución de las tecnologías de la información, la exigencia universal de respetar los derechos humanos, las corrientes icónicas de las industrias globales de la cultura, la política mundial postinternacional y policéntrica, el problema de la pobreza global, los atentados ecológicos globales, el problema de los conflictos transculturales y el terrorismo internacional" (Rodríguez, 2002, p. 446). 


\section{Referencias bibliográficas}

Abraham, M. y Rojas, A. (1997a). La investigación educativa en Iberoamérica. Revista de Educación. 312, 21-42.

Abraham, M. y Rojas, A. (1997b). La investigación educativa latinoamericana en los ultimos diez años. Extraído el 12 de agosto de 2007 desde http:// www.reduc.cl/raes.nsf/0/bfefa77eb2 9a185f04256ac0004f $2 \mathrm{bdf} / \$ \mathrm{FILE} / 00$ 6-42.pdf.

Beck, U. (1998). ¿Qué es la globalización? Falacias del globalismo, respuestas a la globalización. España: Paidós.

Brunner, J. (2000, 23 al 25 de agosto). Globalización y el futuro de la educación: tendencias, desafíos y estrategias. Documento presentado al Seminario sobre prospectiva de la educación en la Región de América Latina y el Caribe, UNESCO, Santiago de Chile.

Castells, M. (2001a). The information Age: Economy, Society and Culture. End of Millennium. Vol. III. Great Britain: Osford Press.

Castells, M. (2001b). The Internet Galaxy. Reflections on the Internet, Business, and Society. New York: Oxford Press.

CONARE. (2006). Plan Nacional de la Educación Superior Universitaria Estatal 2006-2010. Extraído el 12 abril, 2007, de www.conare.ac.cr.

Domínguez, M. (1994, Mayo-Agosto). Perspectivas del desarrollo de la tecnología educativa hacia el año 2000. Revista Iberoamericana de Educación, 5, 67-96.
Mesén, M. (2003). América Latina frente a la desigualdad. En Maestría en Tecnología Educativa: Educación y desarrollo (250). San José, Costa Rica: UNED.

Montiel, K. (2006). Estadísticas básicas sobre la investigación en las instituciones de educación superior universitaria estatal de Costa Rica 2004. San José, Costa Rica: CONARE.

Puryear, J. (2000, Abril). La educación en América Latina: problemas y desafíos. Revista de la escuela de economía y negocio, 4, Año I. Extraído el 20 junio, 2005 de http://www.unsam. edu.ar/unsam/Ediciones/articulos4/ puryear.pdf.

Rodino, A. M. \& Ross, L. (1985). Problemas de expresión escrita del estudiante universitario costarricense: un estudio de lingüistica aplicada. San José, Costa Rica: EUNED.

Rodríguez, M. [Coord.]. (2002). Didáctica general. Qué y cómo enseñar en la sociedad de la información. Madrid: Biblioteca Nueva.

Royero, J. (2005). Las Redes de Investigación y Desarrollo $(I+D)$ en América Latina. Extraído el 12 agosto de 2007 desde http://www.monografias.com/ trabajos32/redes-investigacion/ redes-investigacion.shtml

Ruiz,A. (2006). Universalización de la Educación Secundaria y Reforma Educativa. San José, Costa Rica: Editorial de la Universidad de Costa Rica: CONARE.

Salas, F. (1993). El concepto de Estado en Oscar Arias y Miguel Ángel Rodríguez: Un análisis comparativo. Tesis de Licenciatura para la obtención 
del título de Licenciada en Filosofía, Facultad de Letras. Escuela de Filosofía de la Universidad de Costa Rica.

Schiefelbein, E. (1995). La reforma educativa en América Latina y el Caribe: un programa de acción. Boletín Proyecto principal de educación en América Latina y el Caribe, 37, 3-34.

Vaccarezza, L. (1998, SetiembreDiciembre). Ciencia, tecnología y sociedad: el estado de la cuestión en América Latina. Revista Iberoamericana de Educación, 18, Artículo 1. Extraído el 16 setiembre, 2002 de www.oei.org.

Vizcarro, C. y León, J. A. (1998). Nuevas tecnologías para el aprendizaje. Madrid: Pirámide.

Vygotski, L. S. (1995). Obras Escogidas. Madrid: Visor. 